Reversal of I nt ri nsi c Torque Associ at ed wi th the For mat i on of an I nt ernal Transport Barri er

\begin{tabular}{|l|l|}
\hline $\begin{array}{l}\text { j our nal or } \\
\text { publ i cat i on } \mathrm{t} \text { i t l e }\end{array}$ & Physi cal Revi ew Letter s \\
\hline vol une & Vol .111 \\
\hline number & I ssue5 \\
\hline page range & 055001- 1- 4 \\
\hline year & 2013 08-01 \\
\hline URL & ht t p: //hdl . handl e. net /10655/10170 \\
\hline
\end{tabular}




\title{
Reversal of Intrinsic Torque Associated with the Formation of an Internal Transport Barrier
}

\author{
K. Ida, ${ }^{1}$ H. Lee,${ }^{2}$ K. Nagaoka, ${ }^{1}$ M. Osakabe,${ }^{1}$ C. Suzuki, ${ }^{1}$ M. Yoshinuma, ${ }^{1}$ R. Seki, ${ }^{1}$ M. Yokoyama, ${ }^{1}$ \\ T. Akiyama, ${ }^{1}$ and LHD Experiment Group ${ }^{1}$ \\ ${ }^{1}$ National Institute for Fusion Science, Toki, Gifu 509-5292, Japan \\ ${ }^{2}$ National Fusion Research Institute, Gwahangno 113, Yuseong-gu, Daejeon 305-333, Korea
}

(Received 26 April 2013; published 29 July 2013)

\begin{abstract}
A reversal of intrinsic torque is observed during the formation of an internal transport barrier (ITB) in the Large Helical Device. The intrinsic torque evaluated from the disparity of rotation between coinjection and counterinjection changes sign from counter to codirection inside the ITB after the ITB formation. Both the internal transport barrier and the reversal of the intrinsic torque propagate inwards. This experiment demonstrates that the sign of the nondiffusive term of momentum transport is sensitive to the confinement mode of heat transport.
\end{abstract}

DOI: 10.1103/PhysRevLett.111.055001

PACS numbers: 52.55.Hc, 52.25.Fi, 52.50.Gj

The reversal of intrinsic rotation has been observed in various toroidal plasmas [1-3]. In these experiments, the intrinsic toroidal rotation changes its sign from parallel to the plasma current (codirection) to antiparallel (counterdirection) during the density ramp up or ramp down. The result suggests that the intrinsic rotation and intrinsic torque as a driving force are sensitive to the type of turbulence such as the trapped electron mode (TEM) or the ion temperature gradient (ITG) mode, which is consistent with the change in toroidal rotation during electron cyclotron heating [4-6]. Recently a rotation reversal was observed in the transition regime from saturated ohmic confinement to improved ohmic confinement, which suggests strong coupling between the heat transport and the nondiffusive term of momentum transport [7]. In the Large Helical Device significant spontaneous rotations are observed in the plasmas with an internal transport barrier (ITB) [8] and reversal of spontaneous rotation is observed during density scans [9]. Recently several simulations for the intrinsic torque based on turbulence predict the reversal of intrinsic toque when the dominant turbulence changes [10]. It is not clear how the reversal of intrinsic torque is coupled with a reduction of heat transport and how it propagates radially at the formation of an ITB. In spite of its importance, there are few experimental studies for the evaluation of the intrinsic torque profile [11].

In this Letter, the reversal of intrinsic torque during the formation of an ITB and the radial propagation of intrinsic torque are investigated by the dynamic transport analysis $[12,13]$. In the Large Helical Device the formation of an ion ITB is observed during the decay phase of the electron density after the carbon pellet injection [14]. The ion ITB is characterized by the peaking of the ion temperature profile (not electron temperature profile) in the core region. Figure 1 shows the time evolution of the line averaged electron density measured with a far infrared interferometer, central electron temperature measured with YAG Thomson scattering, central ion temperature and toroidal rotation velocities measured with charge exchange spectroscopy, and toroidal torque driven by neutral beam injection (external torque) in the discharge with neutral beam injection (NBI) in the direction parallel (coinjection) or antiparallel (counterinjection) to the equivalent plasma current. The toroidal torque driven by neutral beam injection (external torque) is calculated from the momentum injection rate from the neutral beam using the FIT-3D code with the correction of the slowing-down process [12].

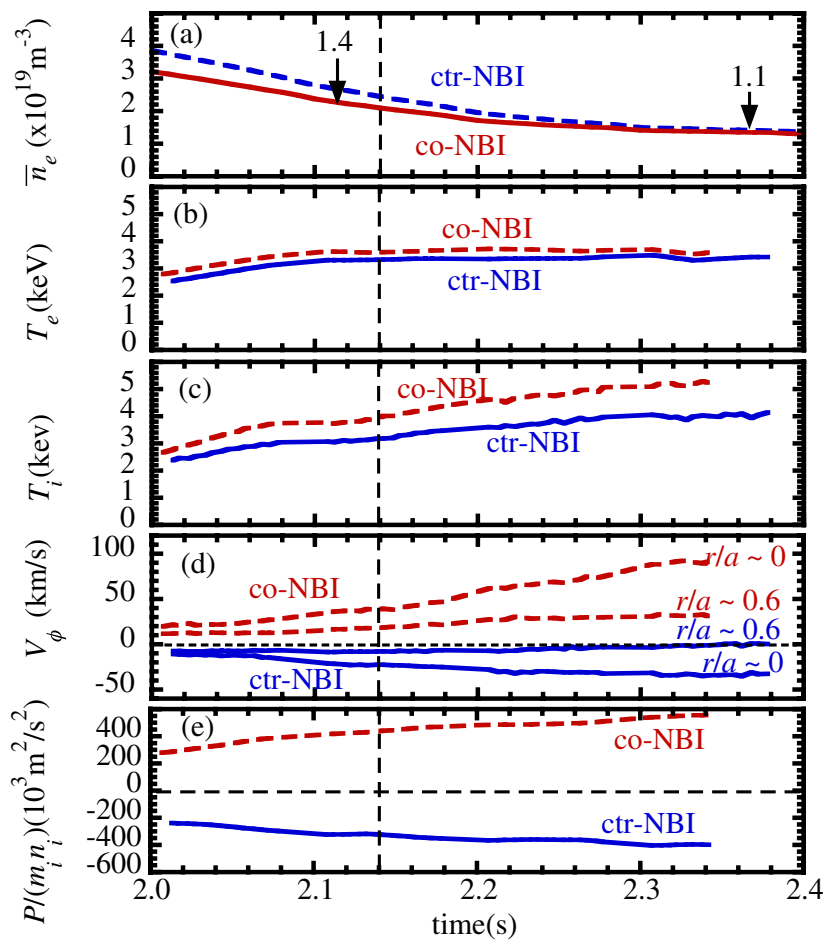

FIG. 1 (color online). Time evolution of (a) line-averaged electron density (density peaking factors, $n_{e}(0) /\left\langle n_{e}\right\rangle$, are also indicated by numbers), (b) central electron temperature, (c) central ion temperature, (d) toroidal rotation velocities, and (e) external torque in the discharge with co-NBI and counter-NBI. 
Although the central electron temperature is constant in time, the central ion temperature starts to increase after $t=2.14 \mathrm{sec}$ during the density decay phase, which indicates the formation of ion ITB. The density peaking factor, $n_{e}(0) /\left\langle n_{e}\right\rangle$, decreases from 1.4 to 1.1 in this phase. There are significant disparities in the toroidal rotation velocity. The toroidal rotation velocity with the co-NBI is much larger than that with the counter-NBI. The toroidal rotation at the normalized minor radius, $r_{\text {eff }} / a_{99}$, of 0.6 is almost zero in the discharge with the counter-NBI, although the external torque of the counter-NBI is comparable to that of the co-NBI. Zero rotation velocity with finite counterexternal torque is clear evidence of intrinsic torque in the codirection.

In this discharge, the formation of an ion internal transport barrier takes place at $t=2.14 \mathrm{sec}$ as the radial heat flux normalized by density increases as seen in the fluxgradient $\left(Q_{i} / n, \nabla T_{i}\right)$ relation in Fig. 2. The slope indicated with the dashed line represents the transport with a constant thermal diffusivity of $\chi_{i}$. There are three phases: increase of $\nabla T_{i}$ with constant thermal diffusivity (phase I), saturation of $\nabla T_{i}$ consistent with a critical temperature gradient model (phase II), and a formation of the ITB (phase III). In phase I ( $t=2.007-2.043)$, the temperature gradients increase linearly as the heat flux increases with a constant thermal diffusivity both inside and outside the ITB. In phase II $(t=2.043-2.102)$, the degradation of transport (increase of thermal diffusivity) is observed both inside and outside the ITB as the temperature gradient increases. This is a transport characteristic which is consistent with the critical gradient transport model, where the temperature gradient tends to be saturated even with the

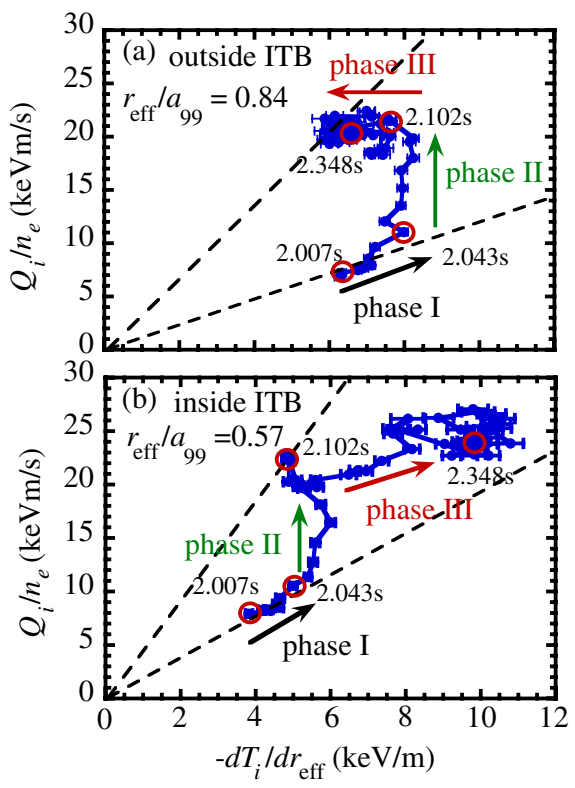

FIG. 2 (color online). Relations between ion heat flux normalized by density and ion temperature gradients at the normalized minor radius of (a) 0.84 (outside the ITB) and (b) 0.57 (inside the ITB). increase of heat flux. When the heat flux increases further, the formation of an ITB starts as indicated with phase III $(t=2.102-2.348)$. In this phase, $\nabla T_{i}$ increases again inside the ITB $\left(r_{\text {eff }} / a_{99}=0.57\right)$, while $\nabla T_{i}$ outside the ITB starts to decrease. It is interesting that the transport degradation (phase II) appears before the transition to the ITB where the normalized heat flux is just below the threshold. The other interesting characteristic is the nonlocal transport feature; simultaneous increase (decrease) of $\nabla T_{i}$ inside (outside) the ITB region.

The time of the formation becomes clearer in the relation between the normalized heat flux and the normalized temperature gradient $(Q / n, \nabla T / T)$ as plotted in Fig. 3. It is interesting that the formation of the ITB is observed only in the ion heat transport, not in the electron heat transport. The normalized electron temperature gradient is almost unchanged even with the large increase of the normalized heat flux by a factor of two. In contrast, the normalized ion temperature gradient increases by a factor of two after the formation of the ITB. The time of the ITB formation can be easily identified by the abrupt increase of the normalized temperature gradient, which is $2.138 \mathrm{~s}$ at $r_{\text {eff }} / a_{99}=0.57$ as seen in Fig. 3(a).

Associated with the formation of the ITB, the intrinsic rotation appears as seen in Fig. 1. Therefore both the reduction of viscosity and the change in the intrinsic torque affect the toroidal rotation as diffusive and nondiffusive terms of momentum transport, respectively. In order to
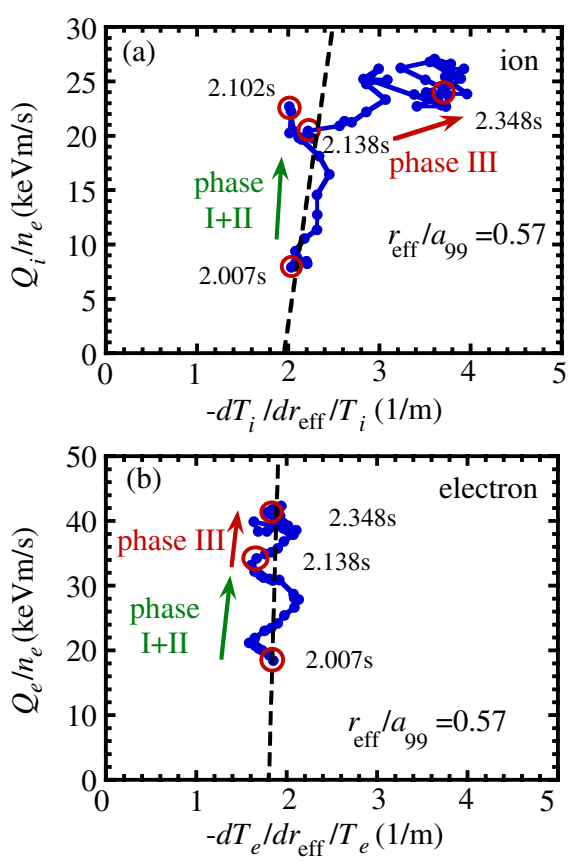

FIG. 3 (color online). Heat flux normalized by density as a function of the inverse of the temperature scale lengths at the normalized minor radius of 0.57 (inside the ITB) for (a) ion and (b) electron heat transport. The dashed lines are guides to the eye for the critical gradient in the $L$-mode plasmas. 
separate the diffusive and nondiffusive terms, the sum and difference of the normalized momentum flux and the velocity gradient between the two similar discharges with ITB except for the direction of the NBI (one is co-NBI dominant and the other is counter-NBI dominant) are derived by the dynamic transport analysis. The momentum flux can be written as $P=-n_{i} m_{i} \mu_{\phi} \nabla v_{\phi}+P_{\mathrm{ND}} . P$ is the momentum flux calculated only from the external torque, and $P_{\mathrm{ND}}$ is the momentum flux due to intrinsic torque. By assuming there is no difference in the perpendicular viscosity, $\mu_{\phi}$ and the nondiffusive term between these two discharges with co-NBI and counter-NBI, one can separate the diffusive and nondiffusive terms as $-n_{i} m_{i} \mu_{\phi}\left[\nabla v_{\phi}(\mathrm{co})-\nabla v_{\phi}(\mathrm{ctr})\right]=P(\mathrm{co})-P(\mathrm{ctr})$ and $2 P_{\mathrm{ND}}=P(\mathrm{co})+P(\mathrm{ctr})+n_{i} m_{i} \mu_{\phi}\left[\nabla v_{\phi}(\mathrm{co})+\nabla v_{\phi}(\mathrm{ctr})\right]$, where $P(\mathrm{co})$ and $v_{\phi}(\mathrm{co})$ are positive for the codirection and negative for the counterdirection. If the external torque input of the two discharges is identical except for the direction, then $P(\mathrm{co})+P(\mathrm{ctr})=0$.

Figure 4 shows the relation between the sum (difference) of the normalized momentum flux and the sum (difference)
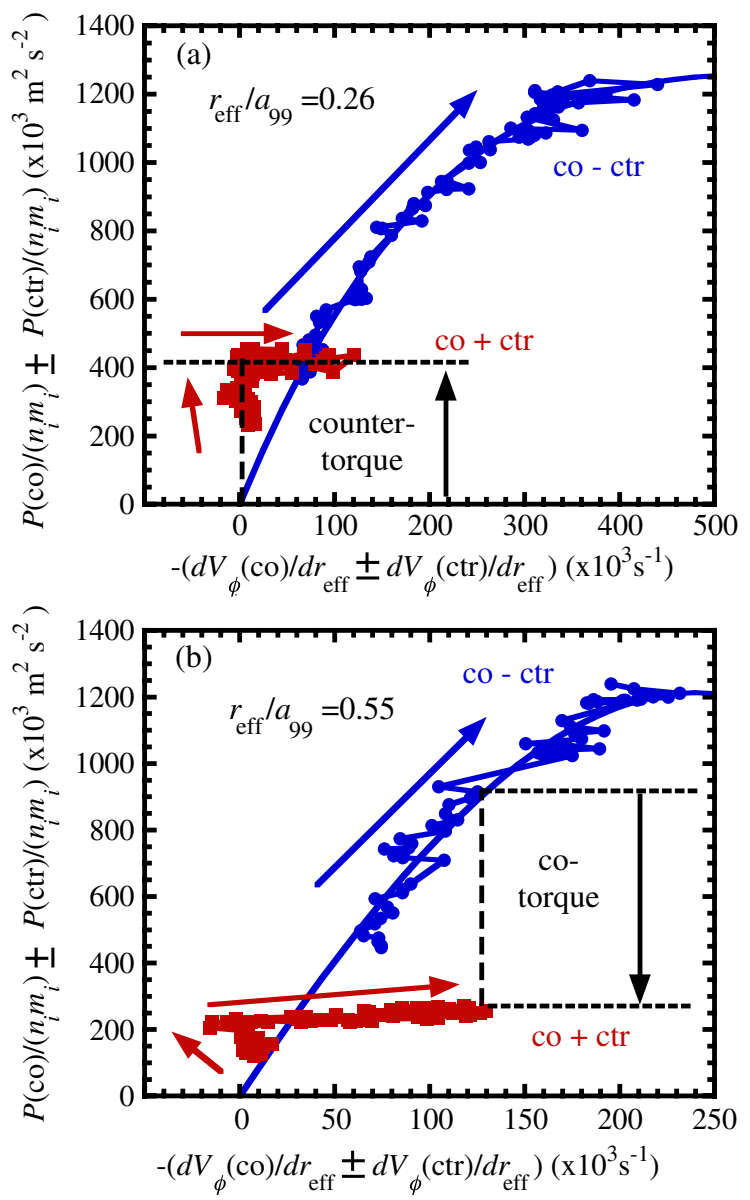

FIG. 4 (color online). Sum (co + ctr) and difference $($ co - ctr) of normalized external torque vs velocity gradient between coinjection and counterinjection discharges at the normalized plasma minor radius of (a) 0.26 , (b) 0.55 . of the velocity gradient. The differences give the relation of the diffusive terms and the sum gives the relation of the nondiffusive term. The relation between the differences shows the diffusive term and the slope gives the viscosity of $\mu_{\phi}$. The viscosity decreases as the velocity gradient is increased after the formation of an ITB as seen in the change of slope of the coctr curve, which is consistent with the reduction of ion thermal diffusivity in phase III seen in Fig. 2(b). When the sum (co + ctr) exceeds the curve derived from the difference (co $-\mathrm{ctr})$, it indicates intrinsic torque in the ctr-direction. On the other hand, it indicates intrinsic torque in the codirection when the sum is below the curve. In the region inside the ITB especially near the foot point $\left(r_{\text {eff }} / a_{99}=0.55\right)$, the intrinsic torque changes direction from the counterdirection to the codirection. The velocity gradient increases in the codirection after the formation of the ITB, which corresponds to the intrinsic torque of $4-5 \times 10^{5} \mathrm{~m}^{2} \mathrm{~s}^{-2}$ in the codirection. The time of the intrinsic torque reversal can be defined as the time when $(\mathrm{co}+\mathrm{ctr})$ cross the curve.

Figure 5 shows the radial profile of the delay time of the ITB formation and the intrinsic torque reversal. The ITB formation is initiated at $r_{\text {eff }} / a_{99} \sim 0.6$ and propagates mainly inwards in the transport time scale of $0.2-0.3 \mathrm{sec}$. The slopes of the time delay of the ITB formations are almost identical between these discharges, which indicates that the propagation characteristic of the ITB does not depend on the direction of the NBI. It is interesting that the ITB formation also propagates outward slightly. There is no reversal of intrinsic torque observed near the magnetic axis of $r_{\text {eff }} / a_{99}<0.3$. The reversal of the intrinsic torque is most significant just inside the ITB $\left(r_{\text {eff }} / a_{99} \sim\right.$ $0.55)$ where the temperature gradient is large enough to produce a residual stress. The radial propagation of the intrinsic torque reversal is slower than that of the ITB formation in the core region. The initial formation of transport barrier for these two discharges takes places at the same time of $2.138 \mathrm{sec}$, although there is some difference in the radial location of the initial ITB formation $\left(r_{\text {eff }} / a_{99}=0.56\right.$ for the co-NBI discharge and 0.67 for counter-NBI as seen in Fig. 5).
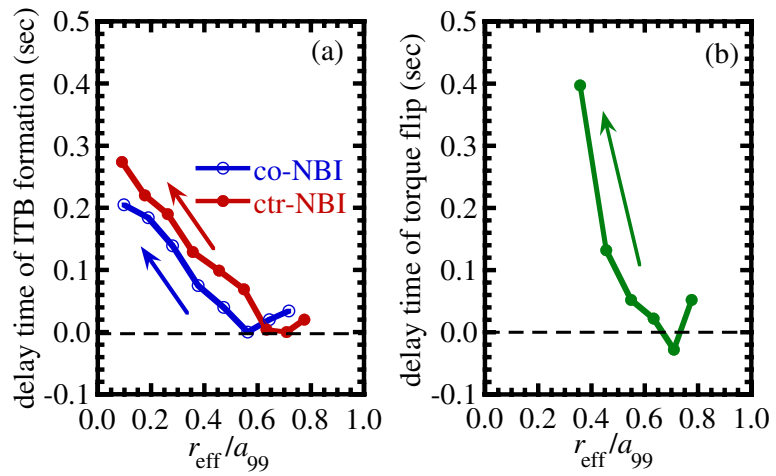

FIG. 5 (color online). Radial profile of the delay time of (a) ITB formation and (b) reversal of intrinsic torque. 


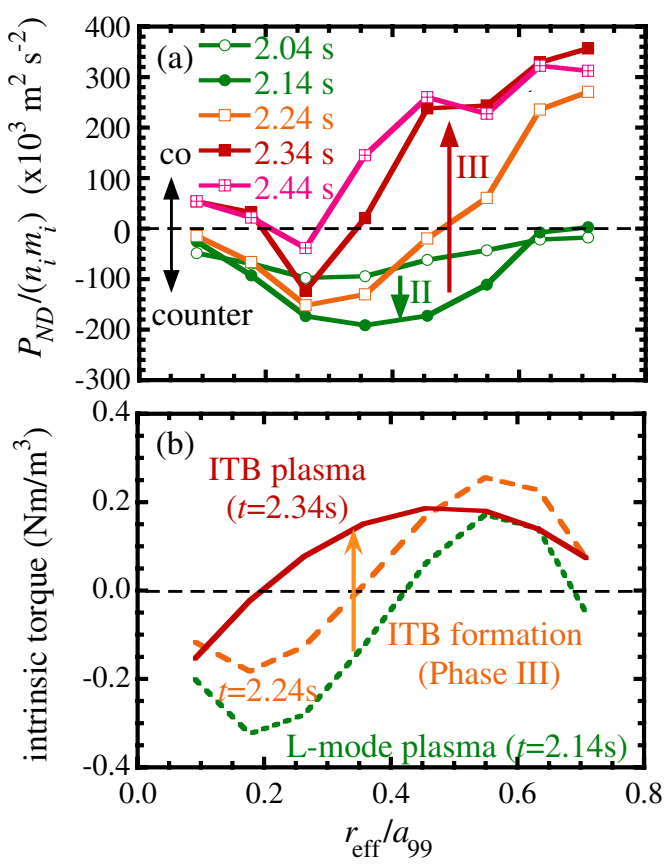

FIG. 6 (color online). Radial profiles of (a) the nondiffusive term of momentum flux and (b) intrinsic torque.

Figure 6 shows the radial profile of the nondiffusive term of the momentum flux estimated from the difference between the (co + ctr) and the curve fitted to the $(\mathrm{co}+\mathrm{ctr})$ data in Fig. 4. Before the formation of the ITB (phase II in Fig. 1), the nondiffusive term of the evaluated momentum flux is negative due to the intrinsic torque in the counterdirection. However, the nondiffusive term of the momentum flux changes sign to positive in the direction to drive the intrinsic rotation in the codirection. This change starts from the edge region and propagates inward. The radial profile of the intrinsic torque evaluated from the nondiffusive term of the momentum flux is plotted in Fig. 6(b). Before the ITB formation, the intrinsic torque is in the counterdirection in the core $\left(r_{\text {eff }} / a_{99}<0.4\right)$, while it is in the codirection in the outer region $\left(r_{\text {eff }} / a_{99}>0.4\right)$. The intrinsic torques in the core region change sign from the counterdirection to the codirection after the formation of the ITB, which clearly shows the strong coupling between the heat transport and the momentum transport. The intrinsic torque in the $L$-mode region is in the counterdirection, while it reverses to the codirection inside the ITB. The reversal of intrinsic torque from counterdirection to codirection is observed to be associated with the formation of ion ITB, where the ITG mode is expected to be unstable and the TEM is considered to be stabilized due to the increase of ion temperature gradient and flattening of electron density profile [15]. This result is in contrast to the reversal of rotation from codirection to counterdirection associated with the transition from the TEM to the ITG mode during the density scan [3].

There are two important features in this experiment. One is that the critical gradient transport feature appears just before the formation of the ITB. The other is the nonlocal response of the transport observed during the formation of ITB. Here, the improvement of transport inside ITB region and the degradation of transport outside ITB region take place simultaneously. The reversal of the intrinsic torque from the counterdirection to the codirection is observed to be associated with the formation of the ITB. The intrinsic torque is in the counterdirection in phase II (degradation of confinement due to critical gradient transport), while it reverses to the codirection in phase III (during the formation of ITB), while the intrinsic torque is always in the codirection near the plasma edge. This experiment demonstrates that the sign of the nondiffusive term of momentum transport is sensitive to the confinement mode of heat transport.

This work is partly supported by a Grant-in-Aid for Specially-Promoted Research (No. 21224014) and for Scientific research (No. 23246164) of MEXT Japan. This work is also partly supported by NIFS10ULHH021.

[1] A. Bortolon, B. P. Duval, A. Pochelon, and A. Scarabosio, Phys. Rev. Lett. 97, 235003 (2006).

[2] J. E. Rice, A. C. Ince-Cushman, M. L. Reinke, Y. Podpaly, M. J. Greenwald, B. LaBombard, and E.S. Marmar, Plasma Phys. Controlled Fusion 50, 124042 (2008).

[3] J. Rice et al., Phys. Rev. Lett. 107, 265001 (2011).

[4] B. P. Duval, A. Bortolon, A. Karpushov, R. A. Pitts, A. Pochelon, and A. Scarabosio, Plasma Phys. Controlled Fusion 49, B195 (2007).

[5] R. M. McDermott, C. Angioni, R. Dux, A. Gude, T. Pütterich, F. Ryter, and G. Tardini, Plasma Phys. Controlled Fusion 53, 035007 (2011).

[6] W.X. Wang, T. S. Hahm, S. Ethier, L. E. Zakharov, and P. H. Diamond, Phys. Rev. Lett. 106, 085001 (2011).

[7] J. Rice et al., Nucl. Fusion 53, 033004 (2013).

[8] K. Ida et al., Nucl. Fusion 50, 064007 (2010).

[9] K. Nagaoka et al., Phys. Plasmas 20, 056116 (2013).

[10] P. H. Diamond et al., Nucl. Fusion 53, 020404 (2013) (to be published in Special issue: FEC2012 Overview).

[11] W. M. Solomon et al., Plasma Phys. Controlled Fusion 49, B313 (2007).

[12] H. Lee, K. Ida, M. Osakabe, M. Yokoyama, C. Suzuki, K. Nagaoka, R. Seki, M. Yoshinuma, and N. Tamura, Plasma Phys. Controlled Fusion 55, 014011 (2013).

[13] M. Yokoyama et al., Plasma Fusion Res. 8, 2403016 (2013).

[14] K. Ida et al., Nucl. Fusion 49, 095024 (2009).

[15] M. Nunami, T.-H. Watanabe, H. Sugama, and K. Tanaka, Phys. Plasmas 19, 042504 (2012). 